\title{
Internal shear cracking in bulk metal forming
}

\section{Christiansen, Peter; Nielsen, Chris Valentin; Bay, Niels Oluf; Martins, P. A. F.}

\section{Published in:}

Institution of Mechanical Engineers. Proceedings. Part L: Journal of Materials: Design and Applications

Link to article, DOI:

$10.1177 / 1464420716681592$

Publication date:

2019

Document Version

Peer reviewed version

Link back to DTU Orbit

Citation (APA):

Christiansen, P., Nielsen, C. V., Bay, N. O., \& Martins, P. A. F. (2019). Internal shear cracking in bulk metal forming. Institution of Mechanical Engineers. Proceedings. Part L: Journal of Materials: Design and Applications, 233(4), 603-514. https://doi.org/10.1177/1464420716681592

\section{General rights}

Copyright and moral rights for the publications made accessible in the public portal are retained by the authors and/or other copyright owners and it is a condition of accessing publications that users recognise and abide by the legal requirements associated with these rights.

- Users may download and print one copy of any publication from the public portal for the purpose of private study or research.

- You may not further distribute the material or use it for any profit-making activity or commercial gain

- You may freely distribute the URL identifying the publication in the public portal 


\title{
INTERNAL SHEAR CRACKING IN BULK METAL FORMING
}

\author{
P. Christiansen ${ }^{1, *}$, C.V. Nielsen ${ }^{1}$, N. Bay ${ }^{1}$ and P.A.F. Martins ${ }^{2}$ \\ ${ }^{(1)}$ Department of Mechanical Engineering, Technical University of Denmark, \\ DK-2800 Kgs. Lyngby, Denmark \\ ${ }^{(2)}$ IDMEC, Instituto Superior Técnico, Universidade de Lisboa, \\ Av. Rovisco Pais, 1049-001 Lisboa, Portugal
}

${ }^{(*)}$ Corresponding author. 


\begin{abstract}
This paper presents an uncoupled ductile damage criterion for modelling the opening and propagation of internal shear cracks in bulk metal forming. The criteria is built upon the original work on the motion of a hole subjected to shear with superimposed tensile stress triaxiality by McClintock (1966) and its overall performance is evaluated by means of sidepressing formability tests in Aluminium AA2007-T6 subjected to different levels of prestrain. Results show that the new proposed criterion is able to combine simplicity with efficiency for predicting the onset of fracture and the crack propagation path for the entire set of test cases regardless the amount of pre-strain derived from previous upsetting under near frictionless conditions. The new proposed criterion can be easily implemented in existing finite element programs and its scope of application allows extending previous work on the opening modes in surface cracking to internal cracks formed under three-dimensional states of stress that are typical of bulk metal forming.
\end{abstract}

Keywords: Bulk metal forming, Internal cracks, Shearing, Ductile damage 


\section{INTRODUCTION}

The utilization of ductile damage mechanics for predicting the initiation and the development of cracks in metal forming has been long discussed from the theoretical and experimental points of view. The different proposed methods and procedures can nowadays be classified into two main categories: (i) coupled approaches based on micro-based damage mechanics (Tvergaard and Needleman, 1984) or built upon continuum damage mechanics (Lemaitre, 1985) and (ii) uncoupled approaches based on the utilization of simple ductile damage criteria that are weighted integrations of the plastic strain (Atkins, 1996).

Micro-based damage mechanics is founded on the microscopic mechanisms of ductile damage processes and is built upon the macroscopic yield surfaces of porous materials (e.g. Gurson, 1977). Continuum damage mechanics combines continuum mechanics and irreversible thermodynamics to replicate damage evolution and the constitutive equations of damaged materials. However, both coupled approaches suffer from limitations in identifying material parameters and properly describing the plastic volumetric strain that are utilized for modelling damage evolution and the associated constitutive equations (Murakami, 2012).

The uncoupled approaches are generally based on the utilization of ductile damage criteria due to Cockcroft and Latham (1968), McClintock (1968) and Bai and Wierzbicki, (2010) among others, and do not account for the progressive deterioration of the material due to the accumulation of damage. However, they are easier to implement in existing finite element computer programs and to define material parameters from experimental data than coupled approaches.

This paper is based on the utilization of uncoupled ductile damage mechanics, which has been extensively utilized in the characterization of surface cracking. Surface cracking is triggered by the accumulation of ductile damage within two-dimensional stress regions (i.e. plane stress regions) that are highly strained due to extensive plastic flow, and its opening 
modes were recently classified into two different groups (Martins et al., 2014): (i) by tension and (ii) by out-of-plane shear, respectively the same as modes I and III of fracture mechanics. Martins et al. (2014) also concluded that the wide applicability of the Cockcroft and Latham (1968) ductile damage criterion results from its capability to predict the onset of surface cracking in opening mode III, which is the most common opening mode found in bulk metal forming. They also confirmed the link between stress-triaxiality $\sigma_{m} / \vec{\sigma}$ (defined as the ratio of the average and the effective stress) and the opening of voids by tension that was originally established by McClintock (1968) and subsequently related to ductile damage criteria for cracking in opening mode I by Atkins and Mai (1985).

The fracture loci associated to the above mentioned uncoupled ductile damage criteria are schematically plotted in the principal strain space (Figure 1a) and in the space of effective strain to fracture vs. stress triaxiality (Figure 1b).

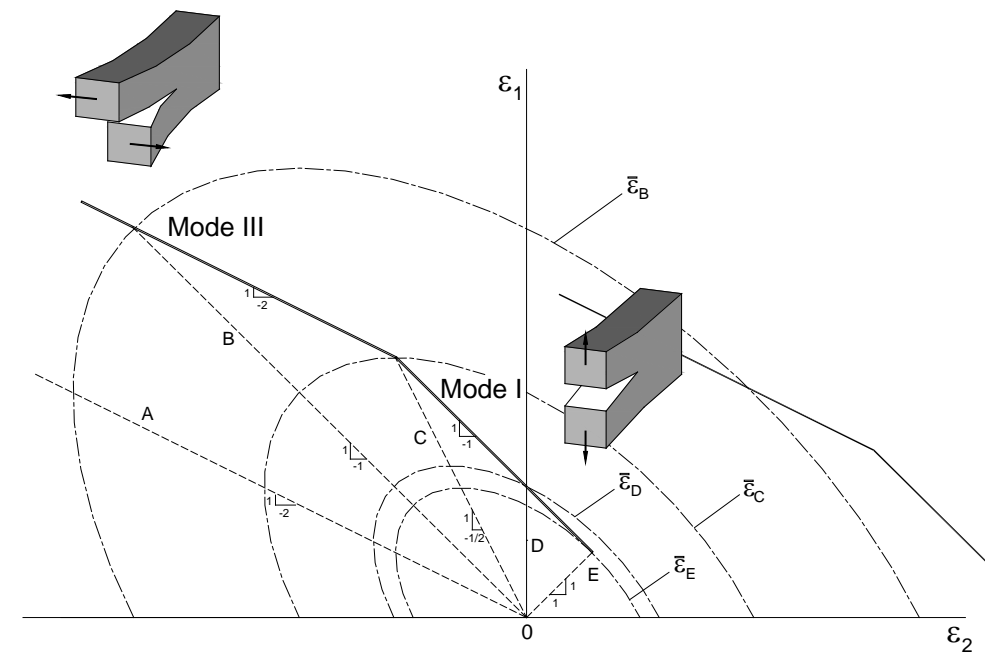

(a)

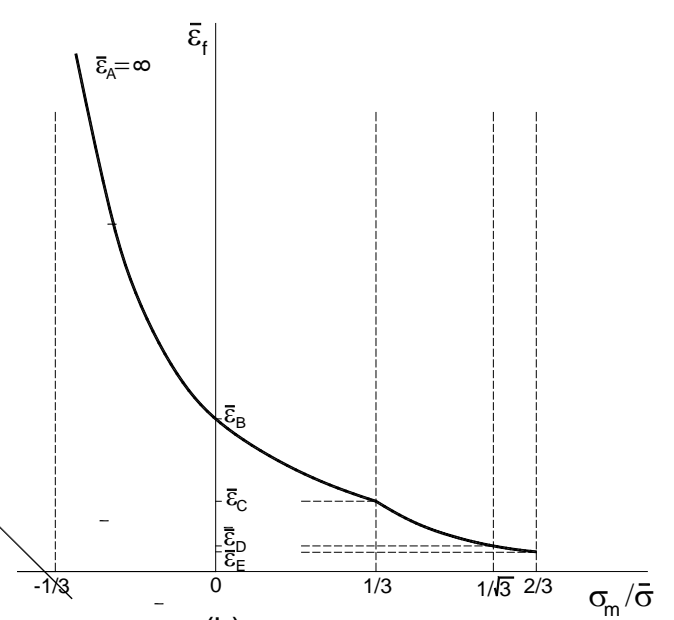

(b)

Figure 1 - Schematic representation of the fracture loci associated to surface cracking in bulk metal forming in the (a) principal strain space and in the (b) space of effective strain to fracture vs stress triaxiality.

In contrast to surface cracking, the research on internal cracking (that is, cracks that are formed under three-dimensional states of stress) has been limited. By internal cracks is meant those initiated inside the workpieces or at the contacting surfaces between material and 
tooling by the opening and subsequent growth of voids. Thus, because the coalescence of these voids into macroscopic cracks takes place by plastic deformation under tension or shear like in case of surface cracking, it is reasonable to expect that the two previously mentioned uncoupled ductile damage criteria for crack opening modes I and III should continue to be successfully utilized in case of internal cracks.

However, the key point is to establish a new ductile damage criterion to characterize the opening and propagation of internal cracks along the high shearing zones (also known as 'shear bands') that are commonly found in bulk forming. In practical terms, and similarly to what Atkins and Mai (1985) did for the opening of voids by tension (mode I), the goal is to develop an uncoupled ductile damage criterion that is capable of modelling the formation of internal cracks by in-plane shear (mode II). As far as the authors are aware, there are no uncoupled ductile damage criteria that have been specifically developed for modelling internal cracks formed by in-plane shear in bulk metal forming.

This paper draws from the original work of McClintock (1966) in the motion of a void subjected to shear with superimposed compressive stress triaxiality $\sigma_{m} / \vec{\sigma}$ and it is aimed at proposing a new uncoupled ductile damage criterion to characterize opening and propagation of internal cracks by in-plane shearing in bulk metal forming. The new proposed criterion is checked against experimental data retrieved from side-pressing tests (Lee and Kobayashi, 1971) without and with pre-straining and the overall performance is compared to that provided by three other well-known uncoupled ductile damage criteria. 


\section{THEORETICAL BACKGROUND}

\subsection{Ductile damage, void coalescence and growth}

Atkins and Mai (1985) worked on McClintock’s (1968) continuum mechanics of void growth in tension to establish the following relation between the inter-void spacing $l$, the diameter $d$ of the void and the stress triaxiality $\sigma_{m} / \vec{\sigma}$ at the onset of cracking (Figure 2a),

$$
\ln \left(\frac{l}{d}\right) \approx \int_{0}^{\bar{\varepsilon}} \frac{\sigma_{m}}{\bar{\sigma}} d \bar{\varepsilon}
$$

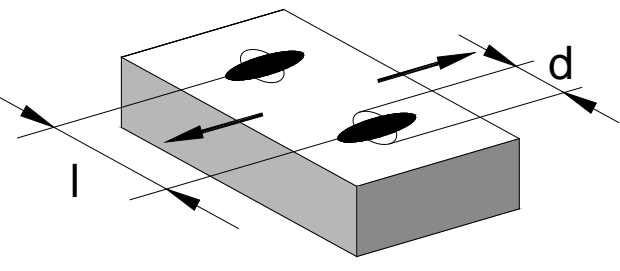

(a)

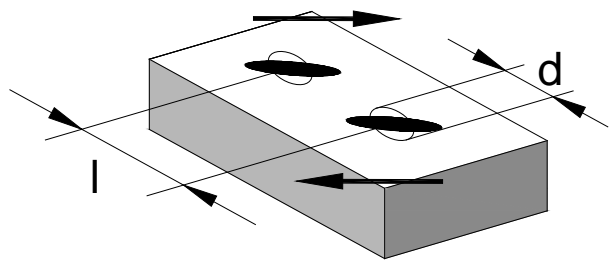

(b)

Figure 2. Schematic representation of void growth in interior cracking by (a) tension and (b) in-plane shear.

Equation (1) relates stress triaxiality to dilatation changes in voids and its right hand side term may be seen as a simplified version of McClintock (1968) ductile damage criterion $D^{t}$ for crack opening by tension (mode I of fracture mechanics),

$$
D^{t}=\int_{0}^{\bar{\varepsilon}} \frac{\sigma_{m}}{\bar{\sigma}} d \bar{\varepsilon}
$$

The value of the critical ductile damage $D_{\text {crit }}^{t}$ is obtained from the material strain conditions $\bar{\varepsilon}_{f}$ at the onset of fracture.

In case of opening and growth of voids by in-plane shear (mode II of fracture mechanics) the starting point is the following relation between the inter-void spacing $l$, the diameter $d$ of 
the void, the strain hardening exponent $n$ and the shear strain $\gamma$ at the onset of cracking (Figure 2b) proposed by McClintock et al. (1966),

$$
\ln \left(\frac{l}{d}\right)=\ln \sqrt{1+\gamma^{2}}+\frac{\gamma}{2(1-n)} \sinh \frac{(1-n) \sigma_{m}}{\tau}
$$

In contrast to equation (1) that is presented in integral form, equation (3) is already the result of integration. This means that it cannot be directly utilized to setup a ductile damage criterion, without additional algebraic manipulation.

A possible solution consists in approximating the first term $\ln \sqrt{1+\gamma^{2}}$ in the right hand side of equation (3) by $\gamma / 3$ for the typical working range of shear strains $\gamma<2$ in cold bulk forming, and simplifying the second term $\sinh \left((1-n) \sigma_{m} / \tau\right) \approx(1-n) \sigma_{m} / \tau$ for typical values of the average and shear stresses in the same order of magnitude. This allows rewriting equation (3) as follows,

$$
\ln \left(\frac{l}{d}\right) \approx \frac{1}{3} \gamma_{f}+\frac{\sigma_{m}}{2 \tau} \gamma_{f}
$$

The required integral form of equation (3) can now be written as,

$$
\ln \left(\frac{l}{d}\right) \approx \int_{0}^{\gamma_{f}} \frac{1}{3} d \gamma+\int_{0}^{\gamma_{f}} \frac{\sigma_{m}}{2 \tau} d \gamma
$$

The above equation is only strictly valid for proportional loading with $\sigma_{m} / \tau$ constant, and considers the opening and growth of voids by in-plane shear to be caused by distortion and dilatation changes. 
Under these circumstances, a new uncoupled ductile damage criterion for internal cracks formed by in-plane shear $D^{s}$ (opening by mode II of fracture mechanics) is proposed by modifying equation (5) as follows,

$$
D^{s}=\int_{0}^{\gamma_{f}} \frac{\tau}{\bar{\sigma}} d \gamma+\int_{0}^{\gamma_{f}} \frac{3}{2} \frac{\sigma_{m}}{\bar{\sigma}} d \gamma
$$

The above modification of equation (5) avoids numerical difficulties resulting from shear stresses $\tau=0$ under homogeneous plastic deformation and the value of the critical ductile damage $D_{\text {crit }}^{s}$ is obtained from the material shear strain conditions $\gamma_{f}$ at the onset of fracture. The first right hand side term of $D^{s}$ is a modified version of the maximum shear strain criterion $\int g d \gamma$, in which $g$ is a non-dimensional weighting function that corrects the accumulated value of the shear strain until fracture as a function of the loading path. In fact, this term can also be seen as a normalized version of the accumulated plastic shear work per volume at fracture $\int \tau d \gamma$. The first right hand side term of $D^{s}$ also provides support to the empirical ductile fracture criterion that was proposed by Martins et al. (2014) to characterize fracture locus by in-plane shear in the principal strain space and in the space of effective strain at fracture vs. stress triaxiality.

The second right hand side term of $D^{s}$ introduces the influence of stress triaxiality $\sigma_{m} / \vec{\sigma}$ in the formation of in-plane shear cracks. This influence was not included in the empirical ductile fracture criterion that was proposed by Martins et al. (2014) and allows concluding that the dependency of the critical ductile damage $D_{\text {crit }}^{s}$ from $\sigma_{m} / \vec{\sigma}$ is different from that of $D_{\text {crit }}^{t}$ because in-plane shear cracks can be formed solely by distortion changes of voids whenever $\sigma_{m}=0$ (that is, in case of pure shear). 
To conclude, it is worth noting that the implementation of the new proposed uncoupled ductile damage criterion $D^{s}$ must not consider the accumulation of negative damage due to dilatational changes when $\sigma_{m}<0$ because the closing up of voids under hydrostatic compression in cold bulk forming does not ensure a recovery of strength due to material healing (suppression of voids) as may be the case in hot bulk forming.

\section{EXPERIMENTATION}

\subsection{Mechanical characterization}

The experimental investigation was carried out in Aluminium AA2007-T6 supplied in the form of rods with $20 \mathrm{~mm}$ diameter. The mechanical characterization of the material at room temperature was carried out by means of standard compression tests in cylindrical test specimens with $20 \mathrm{~mm}$ diameter and $20 \mathrm{~mm}$ height machined from the supplied rods.

The average stress-strain curve resulting from the entire set of experimental data is shown in Figure 3 and its approximation by means of a Voce material strain hardening model is given by equation (7).

$$
\sigma=249+(524-249)(1-\exp (-13.8 \bar{\varepsilon})) \quad(\mathrm{MPa})
$$




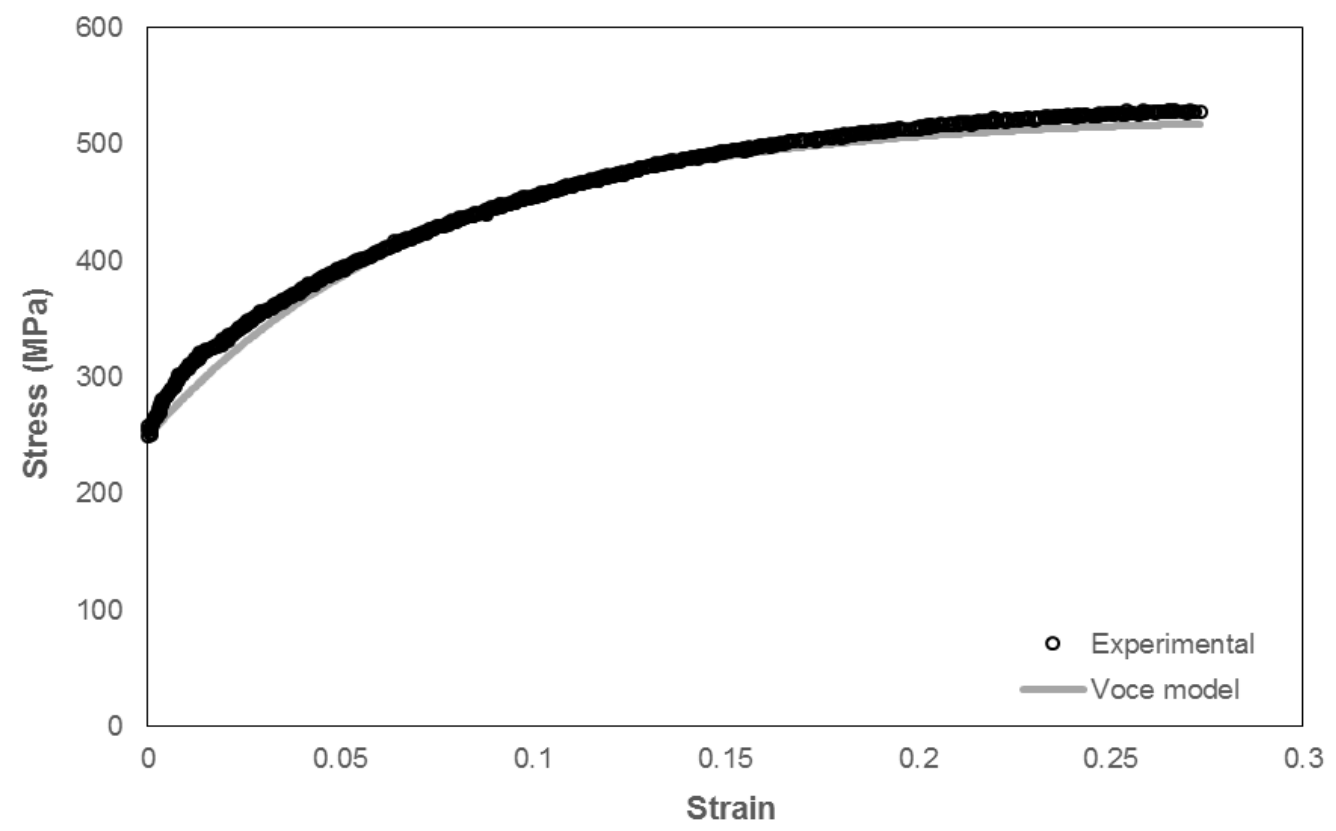

Figure 3. True stress-strain curve of the Aluminium AA2007-T6 obtained by means of compression test.

\subsection{Formability tests}

Side-pressing of cylindrical specimens was the formability test selected to evaluate the overall performance of the new proposed uncoupled ductile damage criterion $D^{s}$ (6). The test is known to provide flow localization along shear bands prior to cracking and consists of compressing the cylindrical specimens between flat parallel platens perpendicular to the cylinder axis of the specimen.

Several cylindrical test specimens with different geometries and pre-strains were utilized (Table 1). The different levels of pre-strain were obtained by upset compression of the cylindrical specimens between flat platens prior to rotation and side-pressing (Figure 4a). The upset compression made use of Teflon sheets on top and bottom ends of the specimens in order to ensure near frictionless conditions. 


\begin{tabular}{|c|c|c|c|c|c|}
\hline \multirow{2}{*}{ Test Case } & \multicolumn{3}{|c|}{ Upsetting } & \multicolumn{2}{|c|}{ Side Pressing } \\
\hline & $\begin{array}{l}\text { Diameter } \\
(\mathrm{mm})\end{array}$ & $\begin{array}{l}\text { Height } \\
(\mathrm{mm})\end{array}$ & $\begin{array}{c}\text { Pre-Strain } \\
\text { (after upsetting) }\end{array}$ & $\begin{array}{l}\text { Diameter } \\
(\mathrm{mm})\end{array}$ & $\begin{array}{l}\text { Height } \\
(\mathrm{mm})\end{array}$ \\
\hline 1 & \multirow{4}{*}{20} & \multirow{4}{*}{20} & - & 20 & 20 \\
\hline 2 & & & 0.11 & 21.1 & 18 \\
\hline 3 & & & 0.21 & 22.2 & 16.2 \\
\hline 4 & & & 0.28 & 23 & 15.1 \\
\hline
\end{tabular}

Table 1. Summary of the experimental formability tests.

The experiments were carried out in a $600 \mathrm{kN}$ Mohr \& Federhaff hydraulic press instrumented with a HBM $500 \mathrm{kN}$ load cell and a HBM transducer for measuring the vertical displacement. Data from both the load cell and the displacement transducer were recorded and treated on a personal computer using LabVIEW data acquisition in order to obtain the force-displacement curves. Examples of the side-pressed test specimens after fracture are shown in Figure 4b.

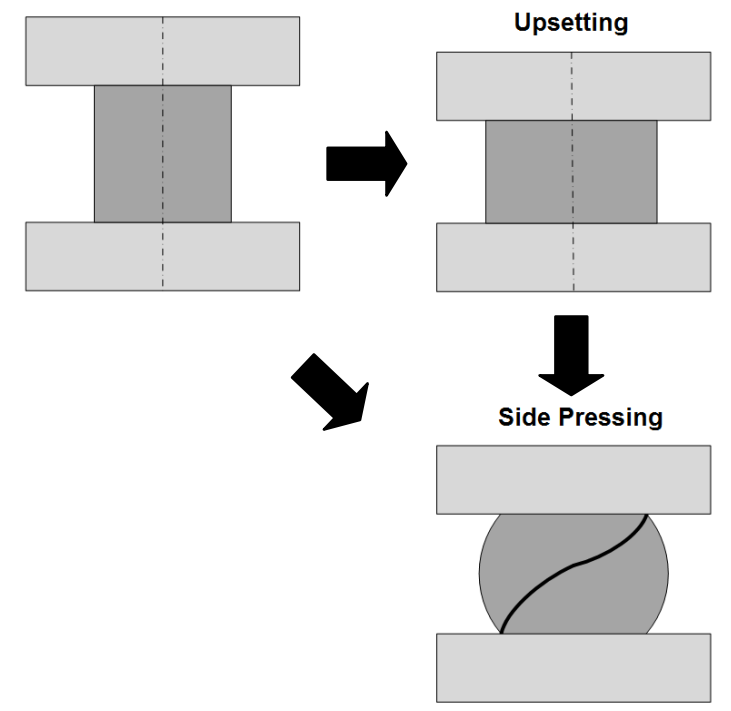

(a)

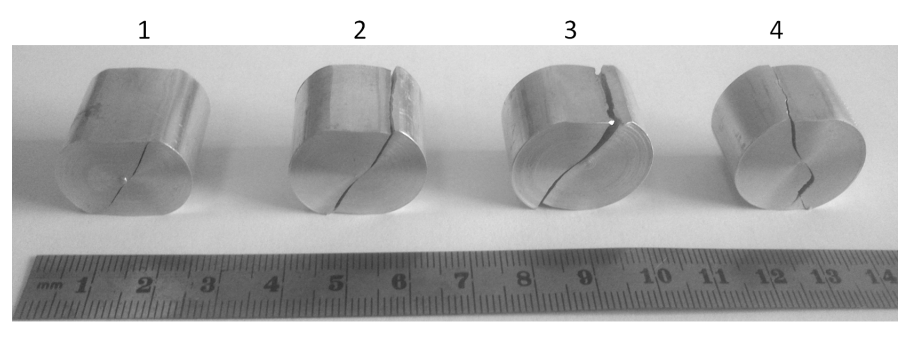

(b)

Figure 4. Side-pressing formability tests: (a) experimental testing methodology and (b) test specimens corresponding to test cases 1 to 4 of Table 1 after fracture. 


\section{FINITE ELEMENT MODELLING}

\subsection{Plastic deformation}

The numerical simulation of the side-pressing formability tests was performed with the finite element computer program I-form. The program was developed by the authors and is built upon the irreducible finite element flow formulation, which is based on the following variational principle (extended to account for frictional effects and contact),

$$
\Pi=\int_{V} \bar{\sigma} \dot{\bar{\varepsilon}} d V+K \int_{V} \dot{\varepsilon}_{v}^{2} d V-\int_{S_{T}} T_{i} u_{i} d S+\int_{S_{f}}\left(\int_{0}^{\left|u_{r}\right|} \tau_{f} d u_{r}\right) d S+P \sum_{c=1}^{N c}\left(g_{n}^{c}\right)^{2}
$$

The symbol $\bar{\sigma}$ in (8) denotes the effective stress, $\dot{\bar{\varepsilon}}$ is the effective strain rate, $\dot{\varepsilon}_{v}$ is the volumetric strain rate, $K$ is a large positive constant imposing the incompressibility constraint by penalization, $T_{i}$ and $u_{i}$ are the surface tractions and velocities on $S_{T}, u_{r}$ and $\tau_{f}$ are the relative velocity and the friction shear stress (according to the law of constant friction $\tau_{f}=m k$ ) on the contact interface $S_{f}$ between tooling and specimens, and $V$ is the control volume limited by the surfaces $S_{U}$ and $S_{T}$. Contact between new surfaces due to crack opening are handled by the last term in (8), which penalizes the normal gap velocity $g_{n}^{c}$ in each of the $N_{c}$ contact pairs by the large positive constant $P$. The normal gap velocity, which is defined as the relative normal velocity between a contacting node and an opposing element segment, is penalized when otherwise leading to penetration.

Further information on the finite element flow formulation and about the computer program I-form can be found elsewhere (Nielsen et al., 2013). 


\subsection{Crack formation and propagation}

The formation and propagation of cracks is modelled by using an element elimination technique in which elements are removed from the mesh when damage $D^{s}$ reaches a critical value. In contrast to element elimination techniques that do not exactly remove the elements from the mesh but simply set to null its stresses and strains in order to stop transmitting forces to neighbouring non-eliminated elements (Wulf et al. 1993), the technique implemented and utilized by the authors removes physically the elements from the mesh. This requires crack propagation to be supported by a node numbering optimization algorithm and by numerical procedures to handle contact with friction between deformable objects in order to account for the interaction between the counterfacing surfaces of the plastically deforming cracks.

Figure 5 shows an example of the crack development between two consecutive steps. Based on the damage criterion proposed in (6), elements will be removed if they exceed the critical damage value. At the same time, and as shown in Figure 5b, the new surfaces may get in contact due to the deformation around the crack. In the present case, an outer compressive stress $\sigma_{C}$ is responsible for the contact between the new surfaces. Numerically, this means that the new surfaces have to be identified and included in the contact search algorithms that will give rice to the contact pairs entering in the last term in (8) to prevent penetration. 


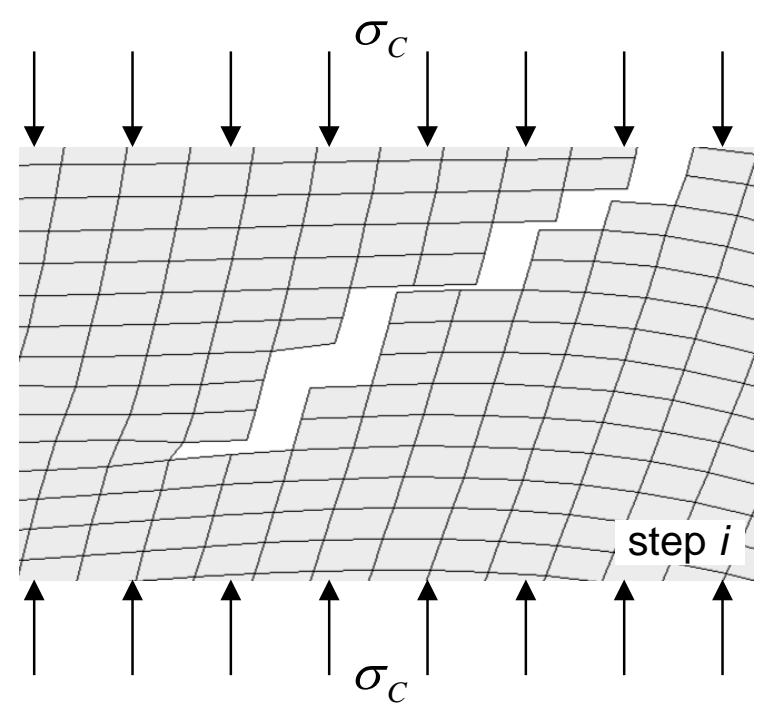

(a)

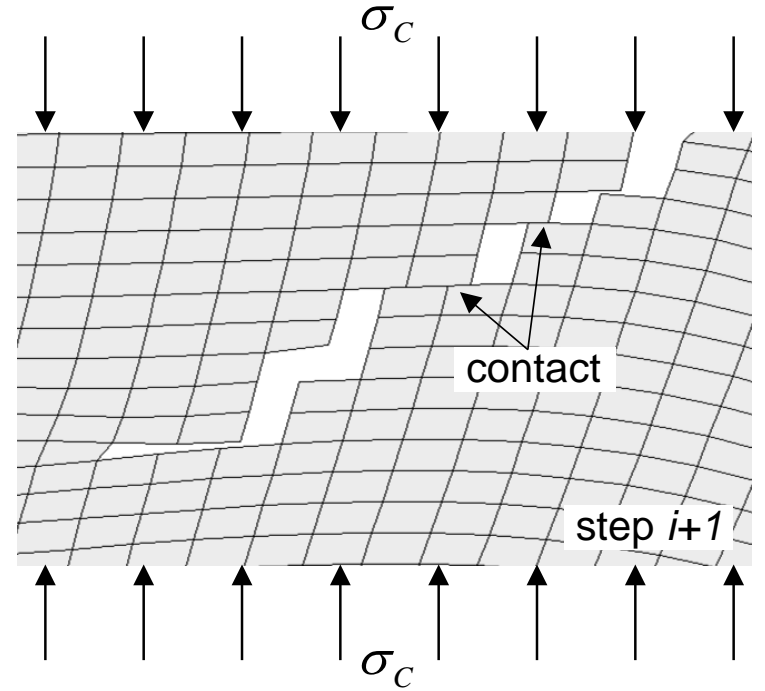

(b)

Figure 5. Finite element mesh with a crack opened by element deletion based on the proposed damage criterion in (6). The situation is shown in case of an outer compressive stress $\sigma_{C}$ resulting in closing of the crack in following steps. Step $i$ in (a) and step $i+1$ (b) show a crack before and just after contact of the two new surfaces.

\subsection{Modelling conditions}

The finite element models utilized in the investigation made use of the near plane stress conditions of the side-pressing cylindrical test specimens. Figure 6 shows a typical finite element model of the cylindrical test specimens before and after side-pressing.

The material cross section is discretized by means of approximately 10000 quadrilateral elements and the upper and lower parallel compression platens are treated as rigid objects and discretized by means of contact-friction linear elements. 


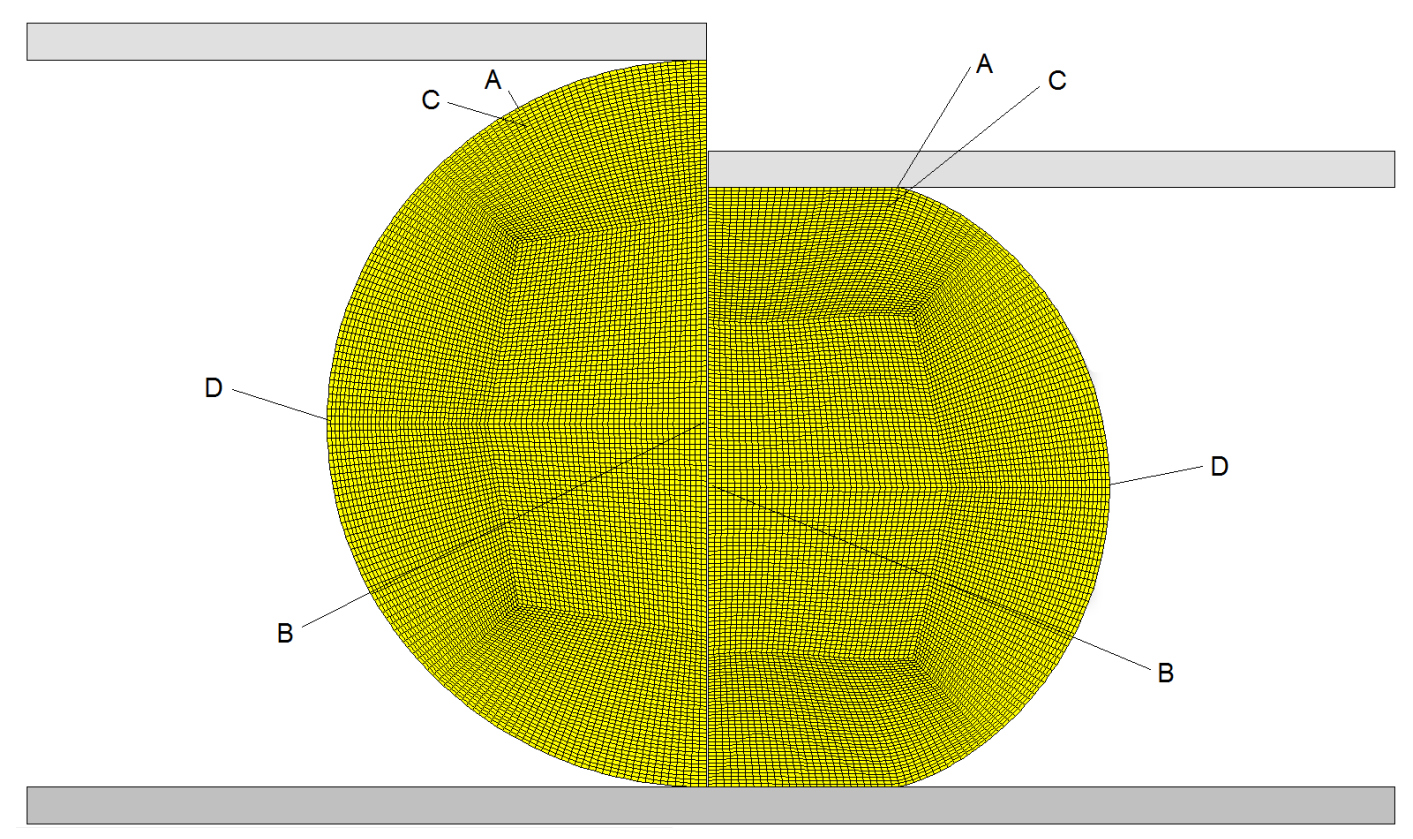

Figure 6. Initial mesh and finite element predicted geometry after side-pressing a cylindrical test specimen with identification of four different locations 'A', 'B', ' $C$ ' and ' $D$ ' that will be latter used in 'Results and Discussion'.

\section{RESULTS AND DISCUSSION}

\subsection{Onset of fracture}

Figure 7 shows the experimental and finite element predicted evolution of the force with displacement for the side-pressing of a cylindrical test specimen machined from the supplied rod that is identified as 'case 1' in Table 1.

As seen in the figure, the force-displacement evolutions show an initial stage (labelled 'S1') characterized by a steep increase of the compression force followed by an intermediate stage (labelled 'S2') in which the force grows at a lower rate up to the onset of fracture, as the contacting area builds up slowly with deformation. The final stage (labelled 'S3') is characterized by a rapid decrease of the force as a result of crack propagation. In fact, crack propagation is so fast in Aluminium AA2007-T6 that it is not possible to stop it and restart at will. 


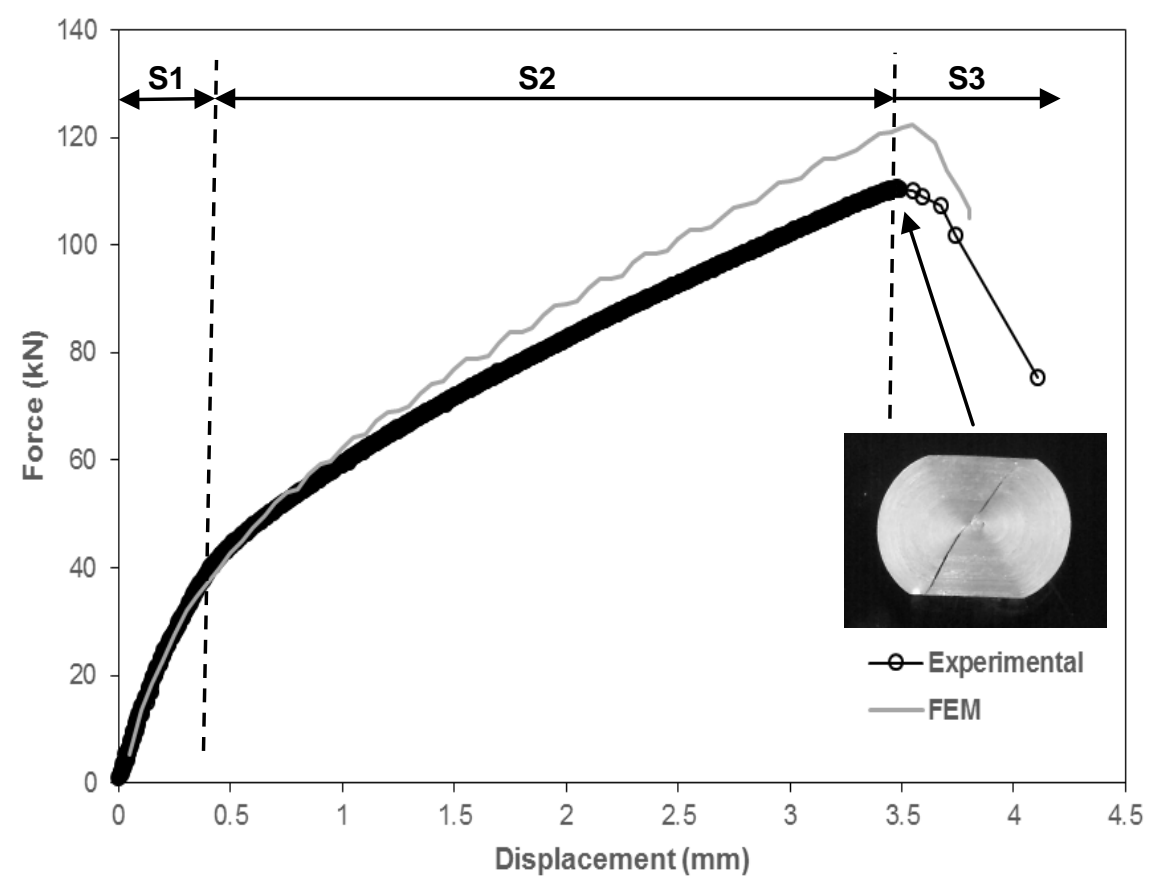

Figure 7. Experimental and finite element predicted evolution of the force with displacement for the sidepressing of a cylindrical test specimen that is identified as 'case 1' in Table 1. The inset picture shows the end face of the test specimen after fracture.

The differences between experimental and finite element predicted evolutions of the force with displacement increase as compression progresses up to a maximum error of approximately $10 \%$ at the onset of fracture. This is attributed to the fact that numerical simulation is performed under simplified plane stress loading assumptions instead of being treated as three-dimensional. However, the plane stress assumption is considered adequate for the purpose of investigating flow localization due to shear strain concentration and subsequent formation and propagation of macroscopic shear cracks by opening mode II.

Figure 8 shows the finite element predicted distribution of accumulated damage at the onset of fracture for case 1 of Table 2 using four different criteria; (a) accumulated plastic strain, (b) normalized Cockcroft-Latham, (c) McClintock's stress triaxiality and (d) the new proposed criterion for in-plane shear based cracking. As seen in the figure, each criterion predicts a different location for the opening of cracks. 
According to the accumulated plastic strain (Figure 8a), for example, cracks should simultaneously occur in a relatively wide region bounded by the contacting surface with the platens and the vertical symmetry line. In contrast, the normalized Cockcroft-Latham ductile damage criterion (Figure 8b) predicts cracks to be formed at the inner central region of the specimen and the McClintock's stress triaxiality based ductile damage criterion (Figure 8c) predicts cracks to be formed at the outer free surface of the specimens close to the platens. All these predictions are wrong because experimental observations allow concluding that cracks are formed at the edges of the upper and lower contacting surfaces with the platens and subsequently propagate along the regions of the cross section in which shear strain concentrates.

However, as shown in Figure 8d, the new proposed criterion $D^{s}$ is capable of predicting the onset of cracking in good agreement with experimental observations. Moreover, its ability to replicate crack propagation along the above mentioned high shearing zones is also very good, as will be shown in the next section. 


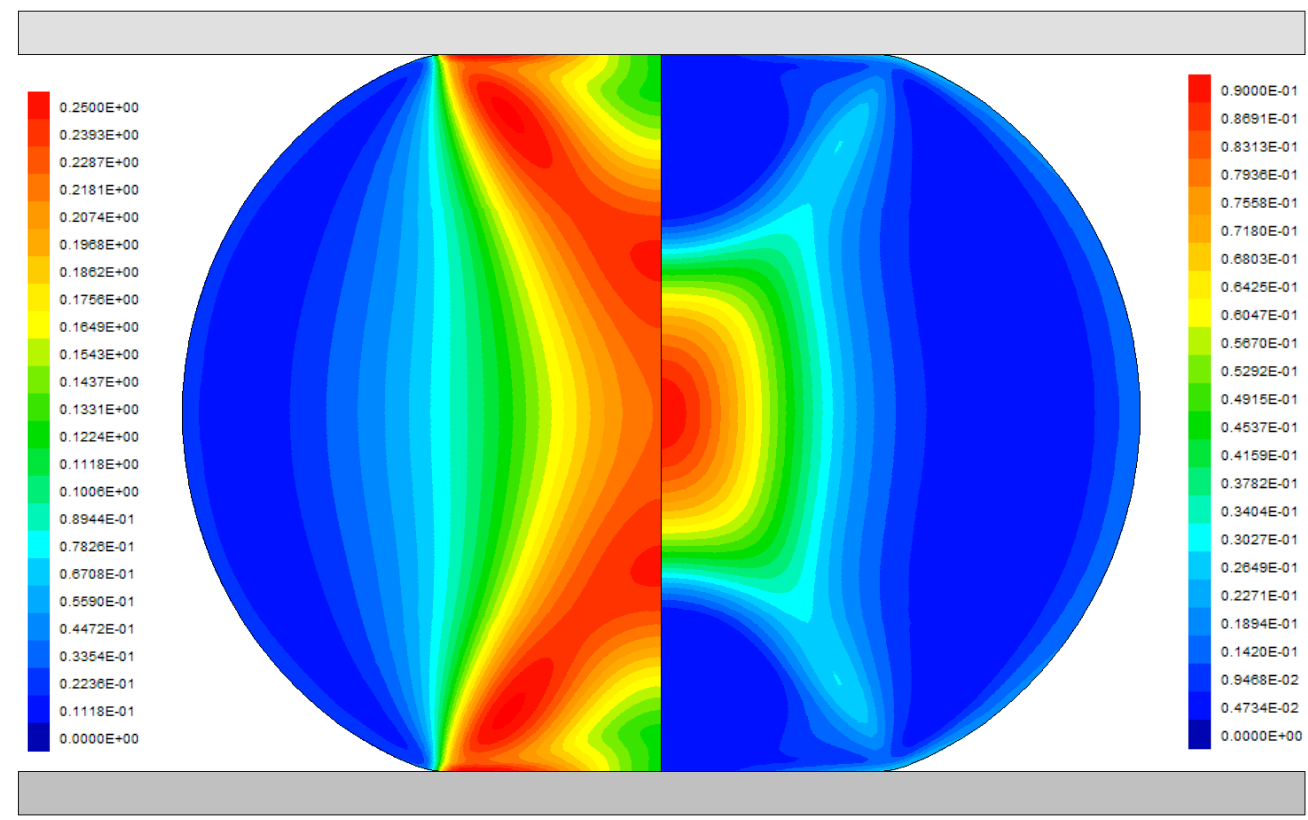

(a)

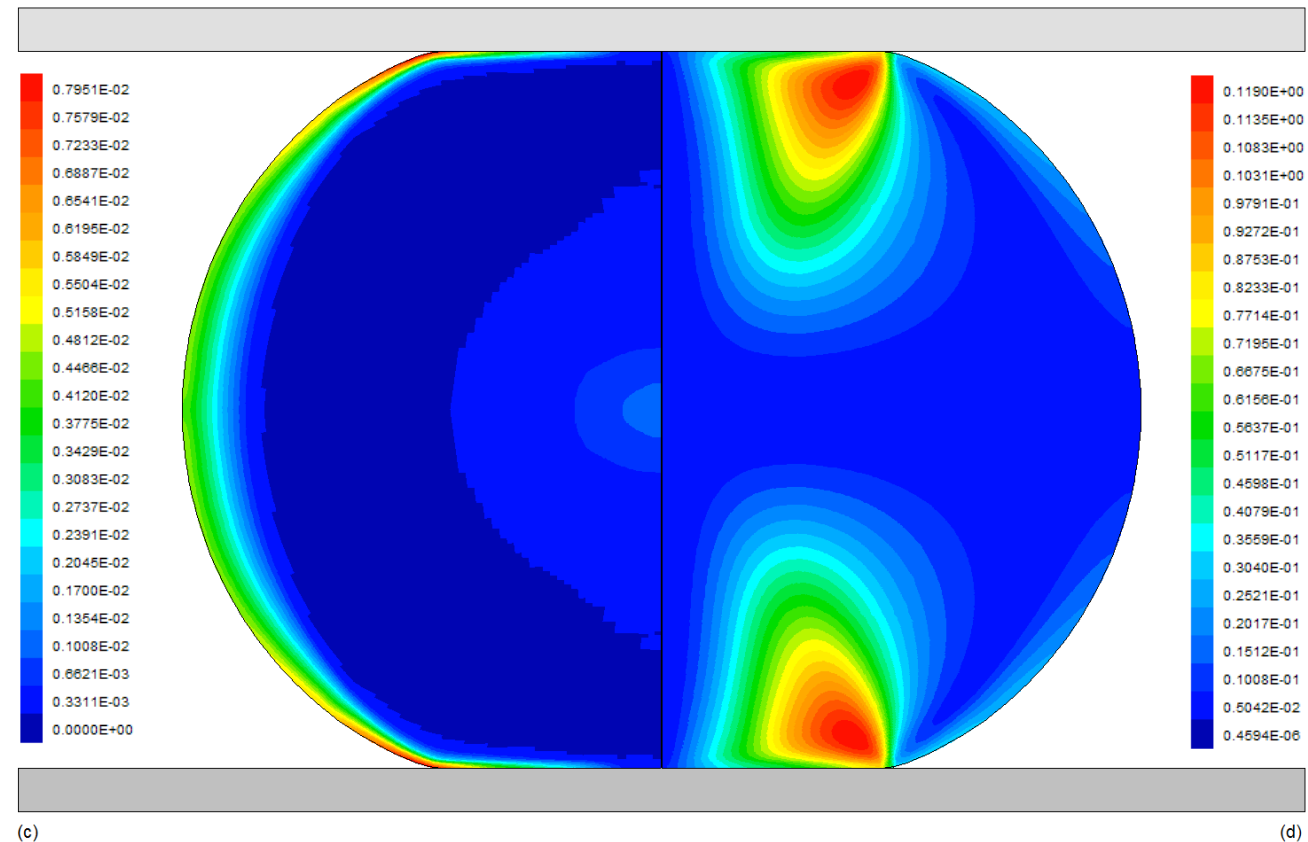

Figure 8. Finite element estimates of the accumulated ductile damage at the onset of fracture for case 1 of Table 1 according to (a) accumulated plastic strain, (b) normalized Cockcroft-Latham criterion, (c) McClintock's stress triaxiality criterion and (d) the new proposed in-plane shear based criterion. 


\subsection{Crack opening and propagation}

An important question regarding the new proposed uncoupled ductile damage criterion $D^{s}$ is to understand if it is capable of providing good estimates of the onset of fracture and of the crack propagation paths in situations where fracture is formed under non-monotonic loading conditions. This paper addresses these two issues by submitting the cylindrical test specimens to a sequence of two different bulk forming operations: (i) upset compression under near frictionless conditions and (ii) side-pressing under dry friction conditions (refer to Table 1).

In what concerns the onset of fracture, experimental observations suggest that crack initiates near the edges of the upper and lower contacting surfaces with the platens regardless the different amounts of compressive side-pressing. According to Figure 9, the largest displacement is obtained for case 1 and the smallest displacement for case 4 , in good agreement with the increasing amount of pre-strain $\bar{\varepsilon}_{0}$ resulting from upset compression (refer to Table 1).
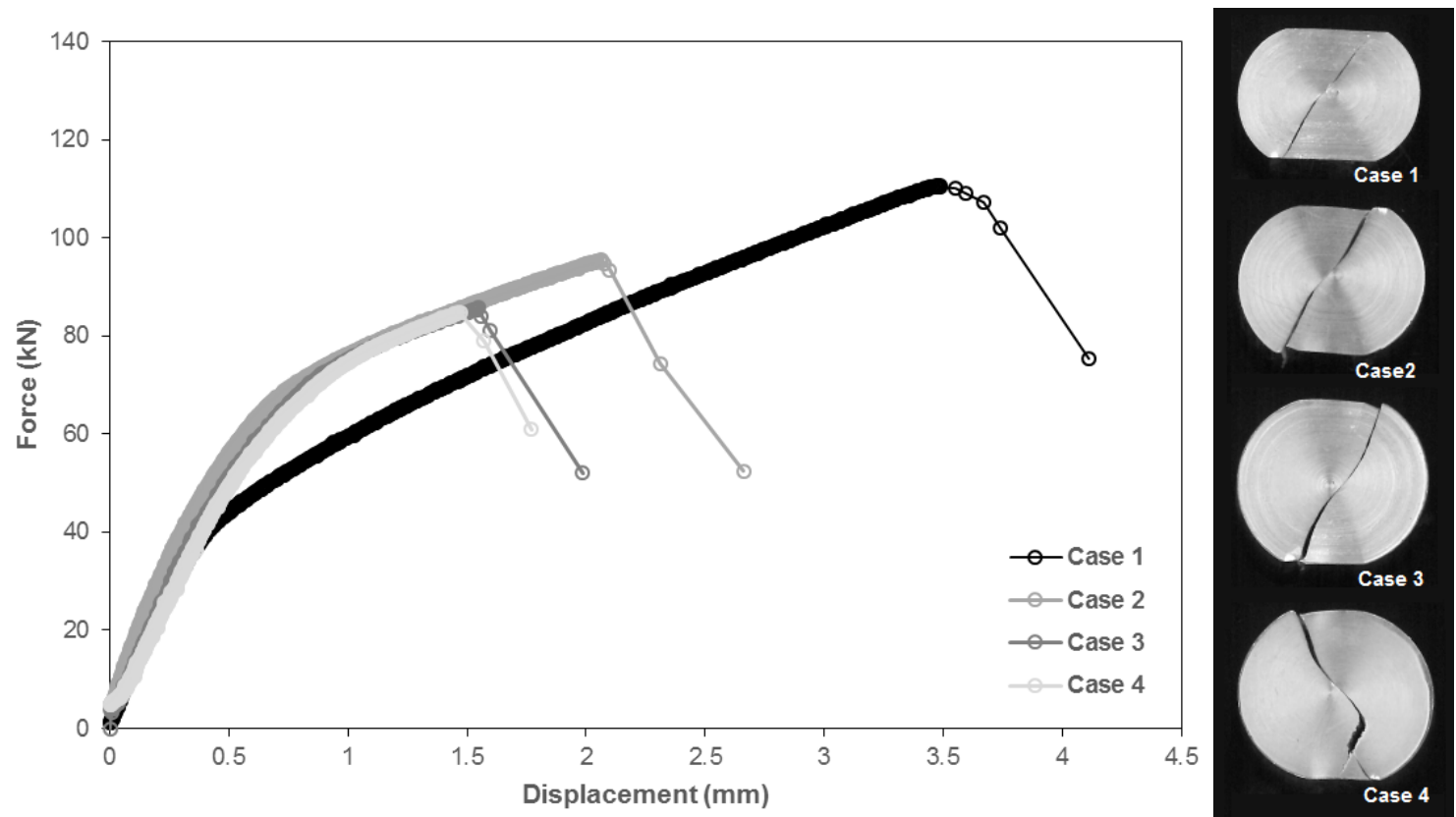

Figure 9. Experimental evolution of the force with displacement for the side-pressing of the cylindrical test specimens of Table 1 . The inset picture shows the cross section of the four different test specimens after fracture. 
By taking the accumulated ductile damage $D_{\text {crit }}^{s}=0.119$ at the onset of fracture for test case 1 (without pre-straining) as critical, the main goal is to determine how good is the agreement between the predicted finite element displacements at fracture and the experimental values taken from Figure 9 for the remaining test cases. The results are summarized in Table 2.

\begin{tabular}{|c|c|c|c|}
\hline Test Case & $\begin{array}{c}\text { Experimental } \\
\text { displacement at fracture } \\
(\mathbf{m m})\end{array}$ & $\begin{array}{c}\text { Finite element predicted } \\
\text { displacement at fracture } \\
(\mathbf{m m})\end{array}$ & $\begin{array}{c}\text { Relative difference } \\
\mathbf{( \% )}\end{array}$ \\
\hline 1 & 3.48 & 3.48 & 0 \\
\hline 2 & 2.07 & 1.93 & 7.25 \\
\hline 3 & 1.55 & 1.75 & -11.4 \\
\hline 4 & 1.47 & 1.80 & -18.3 \\
\hline
\end{tabular}

Table 2. Summary of experimental and finite element predicted displacements at the onset of fracture during side-pressing of cylindrical specimens.

The maximum deviation between experimental and finite element predicted displacements at the onset of fracture is approximately equal to $18 \%$ for test case 4 . However, this difference is probably influenced by the fact that the diameter of the cylindrical test specimen resulting from the preliminary upset compression is not truly uniform as it would be if the process was ideally frictionless.

Figure 10 shows finite element estimates of the accumulated ductile damage at the onset of fracture, of the location where cracks are formed and of its propagation paths for the cylindrical test specimens labelled as cases 1 and 4 in Tables 1 and 2. As seen in the figure, cracks are formed in a subsurface close to the contacting surface (case 1) or at the contacting surface (case 4) between material and platens. The difference between the locations of the onset of fracture is not significant and is attributed to the differences in geometry and prestraining resulting from the upset compression.

The crack propagation paths along the high shearing zones are in good agreement with the experimental observations (refer also to the photographs in Figure 9). Although the element 
elimination technique may suffer from mesh size dependency (meaning that better accuracy could be eventually attained with smaller element sizes), the results shown in Figure 10 are considered good enough to demonstrate the validity of the new proposed uncoupled ductile damage criterion.
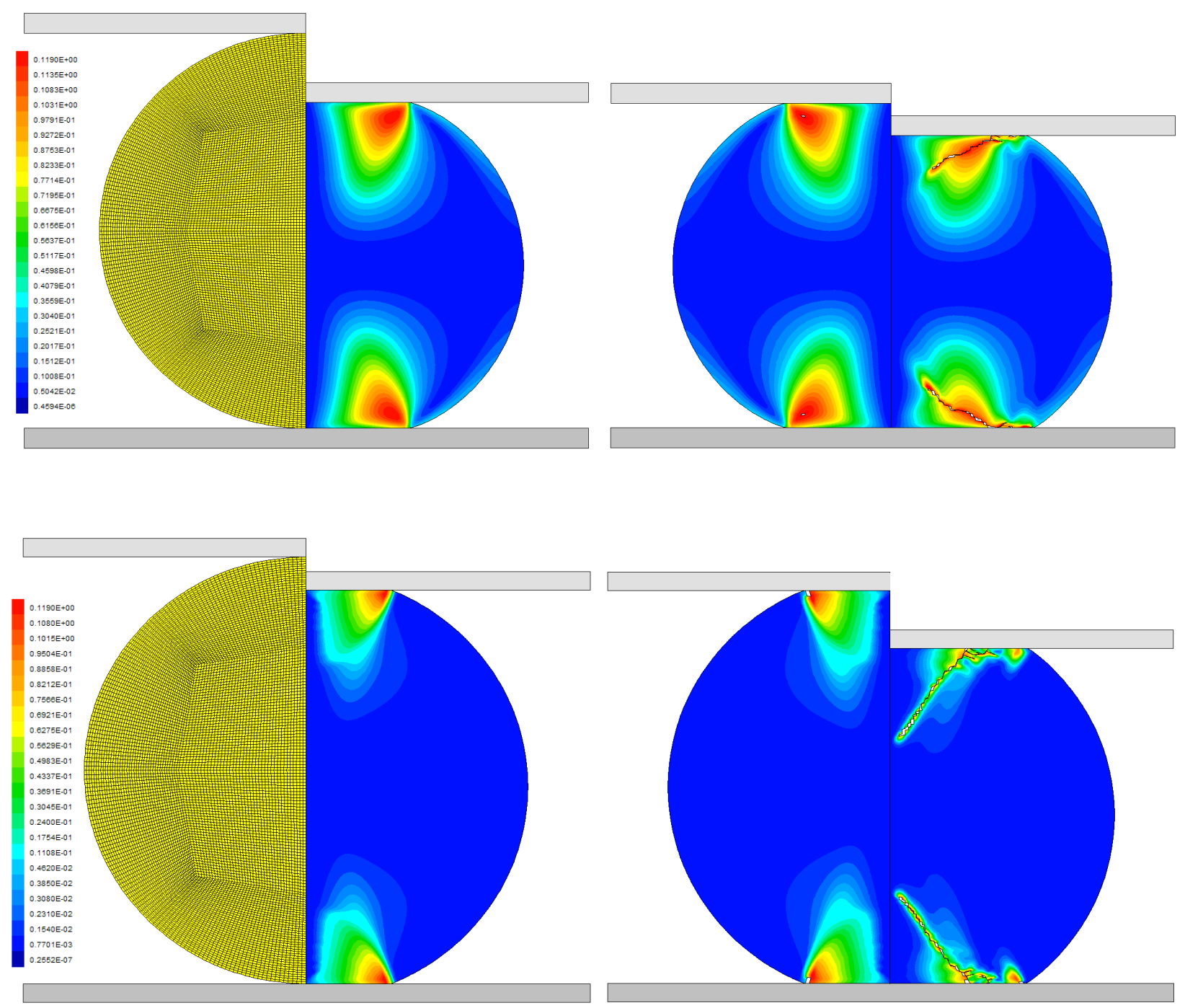

Figure 10. Finite element distribution of accumulated ductile damage and crack propagation for (top) case 1 and (bottom) case 4 of Table 1 according to new proposed ductile damage criterion. 


\subsection{Representation in the space of effective strain vs. stress triaxiality}

Figure 11 presents the loading paths corresponding to locations 'A', 'B', 'C' and 'D' (refer to Figure 6 for identification of these locations) for test cases 1, 2, 3 and 4 of Table 1 in the space of effective strain vs. stress triaxiality.

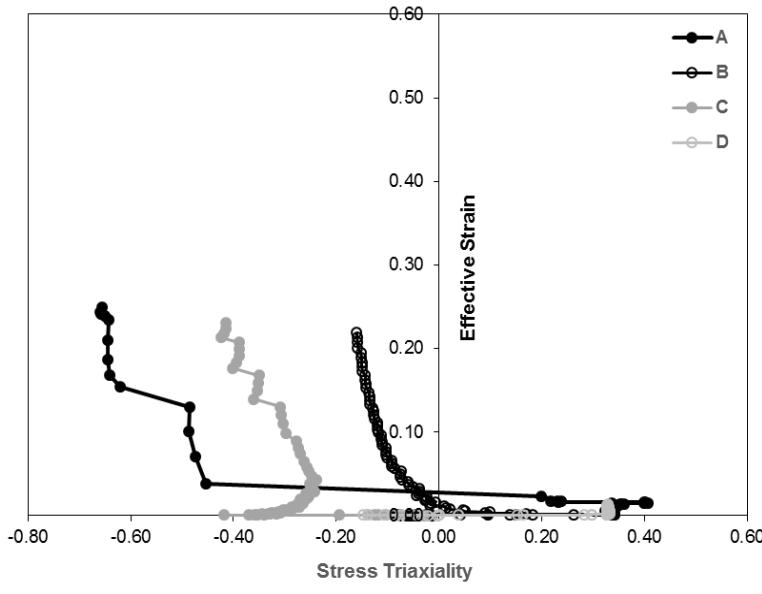

(a)

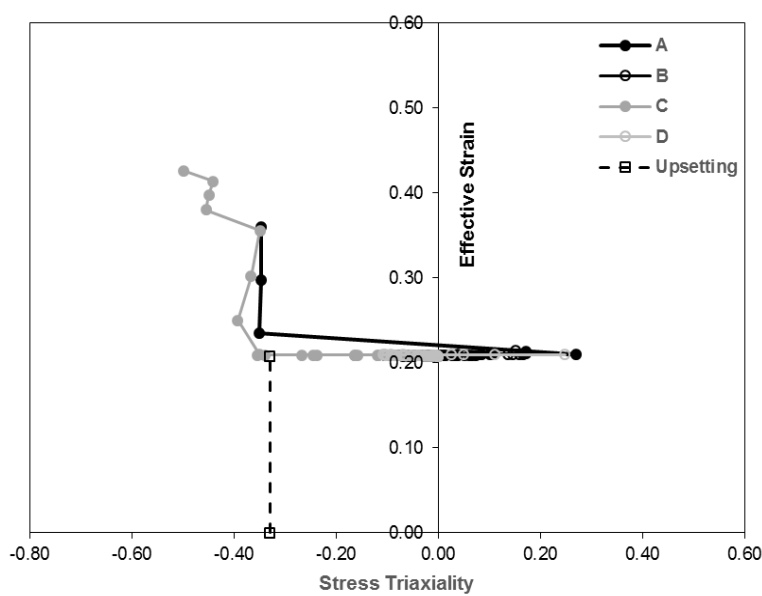

(c)

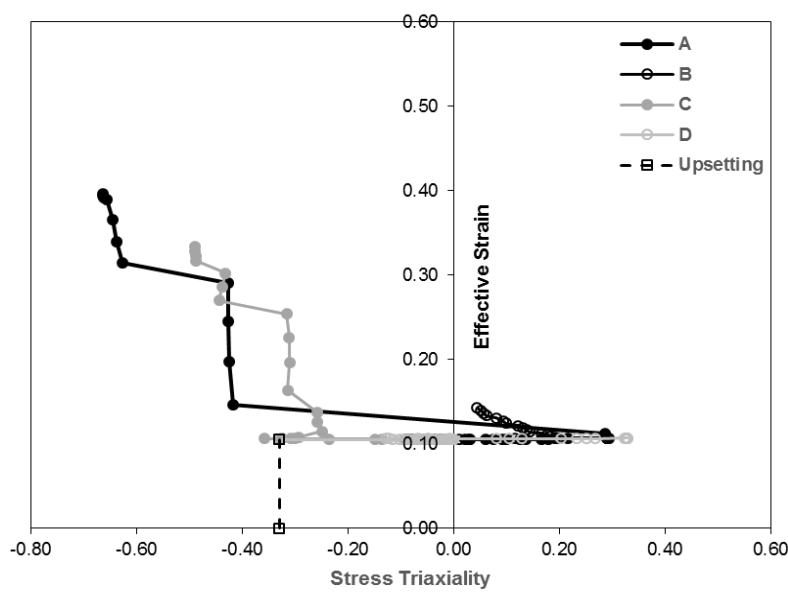

(b)

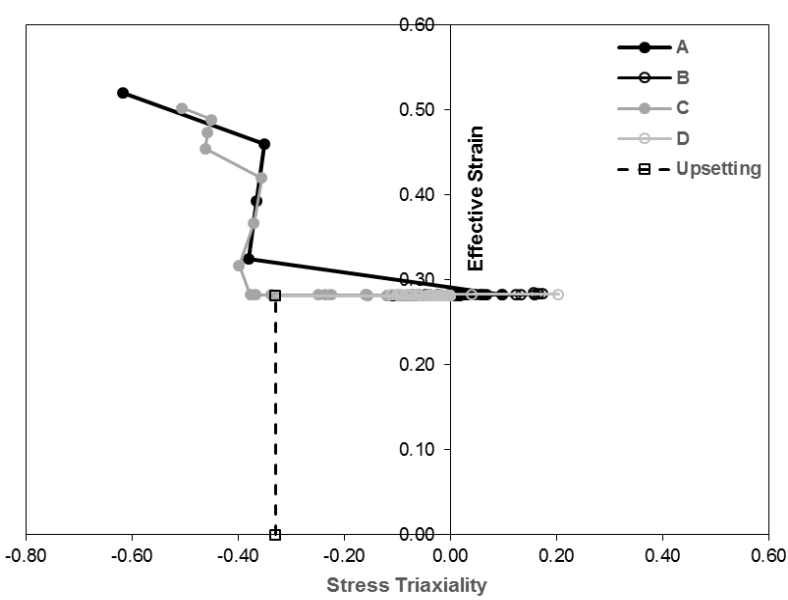

(d)

Figure 11. Representation of the loading paths corresponding to locations ' $A$ ', ' $B$ ', ' $C$ ' and ' $D$ ' (shown in Figure 4) for test cases (a) 1, (b) 2, (c) 3 and (d) 4 of Table 1 in the space of effective strain vs. stress triaxiality.

As seen in the figure, the loading paths corresponding to points ' $A$ ' and ' $C$ ' grow towards the left hand side of the diagrams $\left(\sigma_{m} / \bar{\sigma}<-1 / 3\right)$ as deformation progresses, regardless the amount of pre-strain. The left hand side of the diagrams is located outside the range 
$\sigma_{m} / \bar{\sigma}>-1 / 3$, in which cracks are formed by opening modes I and III (Silva et al., 2015), and may be seen as a region of very high ductility with crack opening modes associated to inplane shearing along localized plastic deformation zones.

However, the finite element predicted loading paths corresponding to points ' $\mathrm{A}$ ' and ' $\mathrm{C}$ ' also reveal the importance of utilizing the space of effective strain vs. stress triaxiality with cautious when modelling multi-stage forming processes like that resulting from upset compression followed by side-pressing. In fact, without subtracting the initial level of prestrain obtained from near frictionless (homogeneous) compression the loading paths of test cases 2, 3 and 4 seem to be moved upwards by a quantity equal to the pre-strain (Figures $11 \mathrm{~b}$ to 11d).

In other words, the initial level of pre-strain needs to be subtracted from Figures 11b to 11c so that the resulting loading paths of ' $\mathrm{A}$ ' and ' $\mathrm{C}$ ' are in good agreement for the entire set of test cases (Figure 12).

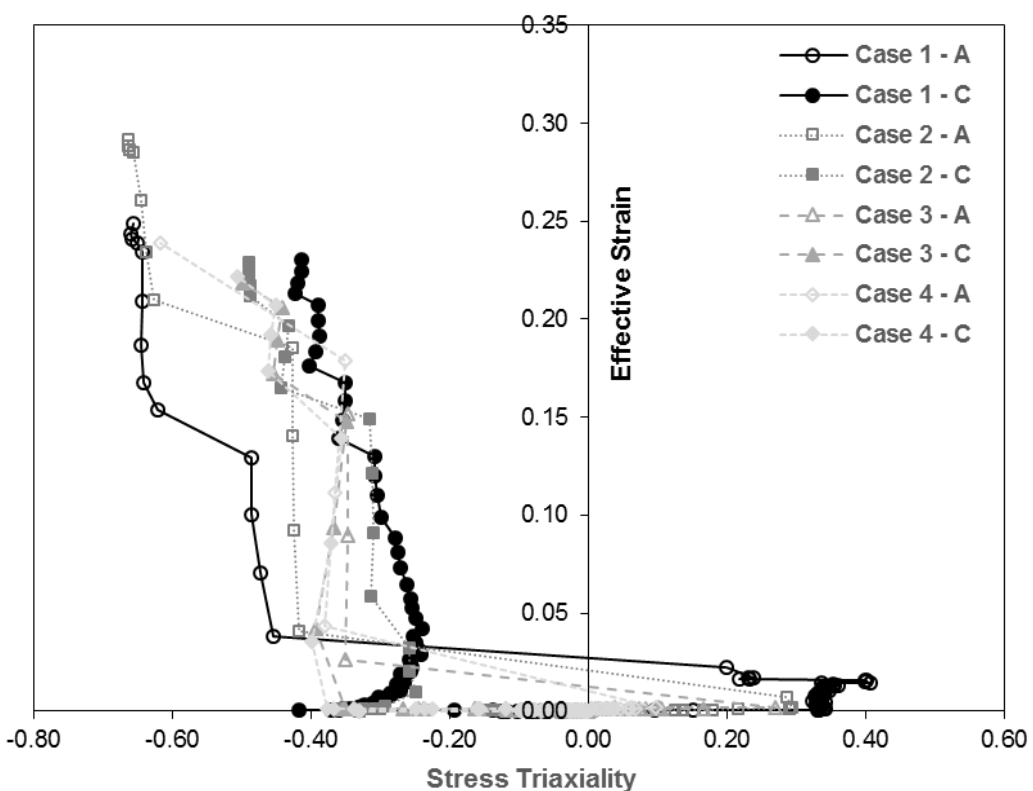

Figure 12. Representation of the loading paths corresponding to locations ' $A$ ' and ' $C$ ' (shown in Figure 4) for test cases 1, 2, 3 and 4 of Table 1 in the space of effective strain vs. stress triaxiality after subtracting the pre-strains obtained by means of frictionless upsetting. 
Regarding locations ' $\mathrm{B}$ ' and ' $\mathrm{D}$ ', their finite element predicted loading paths are found to end at small levels of effective strain in close agreement with the experimental observation that cracks are not triggered in these locations. The only test case in which the final levels of effective strain at ' $B$ ' are of the same order of magnitude of ' $A$ ' and ' $C$ ' is test case 1 (Figure 11a) and the reason for this to happen is because the effective strain in this location is mainly build up from normal strains and not from shear strains which cause failure by internal shear cracking.

\section{CONCLUSIONS}

A new uncoupled ductile damage criterion for modelling the formation and propagation of internal cracks along high shearing zones was developed and applied to side-pressing of cylindrical test specimens made from Aluminium AA2007-T6. The criterion combines accumulation of damage by distortion and dilatation of voids although it can model the growth and coalescence of voids solely by distortion in case of pure shear (that is, when stress triaxiality $\sigma_{m} / \bar{\sigma}$ is zero).

Application of the criterion to side-pressing of cylindrical test specimens with different levels of pre-strain revealed good agreement in predicting the location of the onset of fracture and in replicating crack propagation paths. The suitability of the space of effective strain to fracture vs. stress triaxiality to model the onset of internal cracks was also investigated and the main conclusion is that similarly to the values of critical ductile damage that remain approximately constant for all test cases, the critical loading paths are also very similar if the accumulation of pre-strain under near frictionless upsetting (without damage) is subtracted for each test specimen. 


\section{ACKNOWLEDGEMENTS}

Peter Christiansen and Niels Bay would like to acknowledge the support provided by The

Danish Council for Independent Research under the grant number DFF - 4005-00130.

P.A.F. Martins would like to acknowledge Fundação para a Ciência e a Tecnologia of Portugal and IDMEC under LAETA - UID/EMS/50022/2013.

\section{REFERENCES}

Atkins, A.G., 1996. Fracture in forming. Journal of Materials Processing Technology 56, 609-618.

Atkins, A.G., Mai, Y.W., 1985 Elastic and plastic fracture: metals, polymers, ceramics, composites, biological materials. EllisHorwood, Chichester, UK.

Bai, Y., Wierzbicki, T., 2010. Application of extended Mohr-Coulomb criterion to ductile fracture. International Journal of Fracture 161, 1-20.

Cockcroft, M.G., Latham, D.J. 1968. Ductility and the workability of metals. Journal of the Institute of Metals 96, 33-39.

Gurson, A., 1977. Continuum theory of ductile rupture by void nucleation and growth. I. Yield criteria and flow rules for porous ductile media. Journal of Engineering Materials and Technology - Transactions ASME 99, 2-15.

Lee, C.H., Kobayashi, S., 1971 Analysis of axisymmetrical upsetting and plane-strain sidepressing of solid cylinders by finite element method, Journal of Engineering for Industry (Trans. ASME) 93, 445-454.

Lemaitre, J., 1985. A continuous damage mechanics model for ductile fracture. Journal of Engineering Materials and Technology 107, 83-89. 
Li, Y., Luo, M., Gerlach, J., Wierzbicki, T., 2010. Prediction of shear-induced fracture in sheet metal forming, Journal of Materials Processing Technology 210, 1858-1869.

Martins, P.A.F., Bay, N., Tekkaya, A.E., Atkins, A.G., 2014. Characterization of fracture loci in metal forming. International Journal of Mechanical Sciences 83, 112-123.

McClintock, F.A., Kaplan, S.M., Berg, C.A., 1966. Ductile fracture by hole growth in shear bands. International Journal of Fracture Mechanics 2, 614-627.

McClintock, F.A., 1968. A criterion for ductile fracture by the growth of holes. Journal of Applied Mechanics - Transactions ASME 35, 363-371.

Murakami S., 2012. Continuum damage mechanics: A continuum mechanics approach to the analysis of damage and fracture, Springer, Netherlands.

Nielsen, C.V., Zhang, W., Alves, L.M., Bay, N., Martins P.A.F., 2013. Modeling of thermoelectro-mechanical manufacturing processes with applications in metal forming and resistance welding, Springer, Berlin, Germany.

Silva, C.M.A., Alves, L.M., Nielsen, C.V., Atkins, A.G., Martins, P.A.F., 2015. Failure by fracture in bulk metal forming. Journal of Materials Processing Technology 215, 287-298.

Tvergaard, V., Needleman, A. 1984. Analysis of the cup-cone fracture in a round tensile bar. Acta Metallurgica 32, 157-169.

Wierzbicki, T., Bao, Y., Lee, Y.W., Bai, Y., 2005. Calibration and evaluation of seven fracture models. International Journal of Mechanical Sciences 47, 719-743.

Wulf, J., Schmauder, S., Fischmeister, H.F., 1993. Finite element modelling of crack propagation in ductile fracture. Computational Materials Science 1, 297-301 